\title{
Retour sur la création du colloque « Espace et rapports sociaux de domination : chantiers de recherche »
}

Entretien avec Anne Clerval et Serge Weber

\section{Anne Clerval et Serge Weber}

\section{OpenEdition \\ Journals}

Édition électronique

URL : http://journals.openedition.org/cdg/980

DOI : $10.4000 /$ cdg.980

ISSN : 2107-7266

Éditeur

UMR 245 - CESSMA

\section{Référence électronique}

Anne Clerval et Serge Weber, «Retour sur la création du colloque « Espace et rapports sociaux de domination : chantiers de recherche » », Carnets de géographes [En ligne], 4| 2012, mis en ligne le 01 septembre 2012, consulté le 14 septembre 2020. URL : http://journals.openedition.org/cdg/980

\section{(c) $($ ) $\odot \ominus$}

La revue Carnets de géographes est mise à disposition selon les termes de la Licence Creative Commons Attribution - Pas d'Utilisation Commerciale - Pas de Modification 4.0 International. 


\author{
Retour sur la création du colloque « Espace et rapports sociaux de \\ domination : chantiers de recherche » \\ Entretien avec Anne Clerval et Serge Weber
}

\begin{abstract}
Entretien réalisé le 19 mars 2012 par Marianne Morange - Maître de conférences en géographie à l'Université Paris Diderot et membre du comité scientifique du colloque - avec Anne Clerval et Serge Weber - maîtres de conférences en géographie à l'Université Paris-Est Marne la vallée - co-organisateurs du colloque "Espace et rapports sociaux de domination : chantiers de recherche " organisé par le laboratoire Analyse Comparée des Pouvoirs (EA 3350, Université Paris-Est Marne-la-Vallée) les 20 et 21 septembre 2012 (http://acp.univ-mlv.fr/cerd-2012).
\end{abstract}

M.M : Le colloque que vous organisez s'intitule « espace et rapports sociaux de domination ". Pouvez-vous revenir sur le choix de ce titre ? Qu'est-ce que la notion de domination recouvre pour vous?

A.C: L'idée, c'était de réinterroger une entrée anticapitaliste et marxiste sur les rapports de classes et de domination mais nous ne voulions pas nous centrer uniquement sur les rapports de classes. Nous voulions inclure les autres types de domination, classiquement les rapports de sexe, de race... et éviter un titre trop vague, en parlant par exemple de "géographie critique ". Nous voulions que ce soit très précis, sur les rapports sociaux de domination, de classes, de races, de sexes, selon une approche matérialiste. C'est-à-dire que nous considérons que chacun de ces rapports est fondé sur une exploitation matérielle. C'est ce qui figurait en filigrane dans l'appel, même si cela n'a pas été clair pour tout le monde et qu'il nous a parfois fallu réexpliciter ce que nous entendions.

S.W : Je dirais que par rapport à beaucoup de champs critiques, par exemple dans les approches culturelles, de genre, ou dans le champ des études migratoires d'où je viens, j'ai souvent remarqué que même dans des études très pertinentes et critiques, on oubliait l'intérêt que les classes dominantes, ou des groupes dominants et des individus qui ont des positions dominantes, ont à l'interaction. II s'agit de rappeler que les inégalités sont le produit d'interactions.

A.C: Oui, le produit de rapports de pouvoir. Ce n'est pas juste une question de stratification: "il y a des plus riches et des plus pauvres ». C'est parce qu'il y a des gens qui dominent qu'il y a des gens qui sont dominés; les deux vont ensemble. On veut promouvoir une vision pas seulement descriptive, mais qui porte à la fois une description et une cause, qui explique. 
S.W : Et je pense que c'est intéressant de voir ce qu'il y a pour certains de profit à tirer des rapports de domination.

M.M : Vous avez donc délibérément évité le terme "critique " dans le titre du colloque, mais il apparaît dans l'appel. Pouvez-vous $\mathrm{m}$ 'expliquer pourquoi et comment vous l'articulez à la notion de domination, comment vous positionnez le colloque dans le champ des pensées critiques?

S.W : La pensée critique c'est très large, il y a beaucoup de façons de faire de la critique. On parle de critique post-moderne, de critique foucaldienne, de critique queer; on peut même faire une critique lacanienne. Le projet risquait de rester trop large.

A.C: Je dirais que le champ critique est très vaste dans ses approches et que nous voulions quelque chose de plus précis que cela. Dans l'appel, on a utilisé le terme critique quand on s'inscrivait dans un champ, celui du renouveau de la pensée critique, ou pour parler de la géographie critique; et quand on parlait du colloque, on parlait soit de rapports sociaux de domination, soit de géographie radicale. On trouvait ça pratique de parler de géographie radicale pour aborder la question des rapports sociaux de domination, pour parler d'une géographie centrée sur les rapports sociaux de domination, même si je ne suis pas sûre que la géographie radicale américaine se définirait comme cela. Mais dans la géographie radicale américaine, il y a au moins cette entrée marxiste, même si elle n'est pas la seule, et elle aborde les rapports dominants-dominés. Cela dit, inversement, par rapport à $D$. Harvey qui ne prend pas en compte les autres rapports de domination, on voulait aussi avoir les apports de ces différentes approches. C'est vrai que les deux termes figurent dans l'appel: pour désigner le champ plus large dans lequel s'inscrit le colloque, et un aspect spécifique de ce champ que nous voudrions approfondir avec les participants au colloque.

M.M : On parle de renouveau des pensées critiques en France. Qu'en pensez-vous ? Avez-vous le sentiment que ce colloque participe de ce renouveau ?

S.W : Oui, bien sûr ! On le dit dans l'appel.

A.C : Mais pour moi, ce renouveau est récent. Enfin, en géographie, il n'est visible que depuis peu de temps. II y a à la fois des racines anciennes, qui ne se sont jamais interrompues, et une certaine effervescence, comme la revue Justice Spatiale, toute récente me semble-t-il ${ }^{1}$. Il y a une envie, une visibilité, et des gens qui commencent à se dire qu'il se passe quelque-chose, même si ça ne veut pas dire qu'il ne se passait strictement rien avant.

S.W : Je dirais qu'à une certaine époque, certains géographes étaient ouvertement marxistes, ce qui n'avait pas le même sens qu'aujourd'hui. C'était un positionnement,

\footnotetext{
${ }^{1}$ Justice Spatiale / Spatial Justice, revue pluridisciplinaire et bilingue française (français-anglais) dédiée à l'étude des relations entre justice et espace, créée en 2009 suite au colloque "Justices et injustices spatiales », Paris X-Nanterre 2008 (http://jssj.org/).
} 
je ne dirais pas dominant, mais tout de même représenté par de grandes figures. Aussi bien en France qu'en Allemagne ou dans d'autres pays, ce positionnement a été discrédité pour des raisons purement politiques, et les gens qui les ont critiqués l'ont fait à une époque marquée par la Guerre froide et l'anticommunisme qui se traduisait parfois par une méfiance vis-à-vis de l'interprétation marxiste dans les sciences humaines. Et finalement, à un certain moment, ça a disparu. On a parlé d'autre chose, et les intéressés eux-mêmes ont eu du mal à continuer. Et ensuite, d'autres pensées critiques ont émergé, par exemple vis-à-vis de l'État-nation sur la dimension transnationale en ce qui concerne les migrations, ou les courants qui faisaient une part aux stratégies individuelles, aux résistances; et avec ça, ce qui a disparu, c'est l'ancrage marxiste ou marxien. Finalement, en géographie, c'est surtout dans les études sur le Sud, les migrations, la ville que beaucoup de gens ont eu des positions ouvertement critiques et matérialistes; mais c'est venu de manière atomisée, dispersée.

A.C : Et sur la ville, c'est un petit courant, en réalité, qui fait le lien avec des systèmes de pouvoir. Même aux États-Unis où on entend beaucoup parler d'Harvey, c'est quand même marginal parmi ceux qui travaillent sur la ville. Et sur la ville, il y a aussi eu beaucoup de choses en aménagement du territoire et en urbanisme, assez lénifiantes, liées aux technocrates, et même s'il y a des éléments critiques dans ces approches, elles occultent les rapports de pouvoirs, comme si ce n'était pas possible, notamment pour la DATAR, de publier ce genre de choses.

S.W : Je pense que c'est aussi lié aux difficultés de la recherche, à la baisse des financements, à celle des effectifs étudiants, à l'incertitude pour beaucoup d'enseignants-chercheurs et de chercheurs en géographie. Beaucoup de départements se sont dits "il faut qu'on survive " et ce sont les catégories mainstream qui ont été adoptées: aménagement, développement durable... et pendant une période, ces approches sont devenues dominantes.

A.C : Elles dominent encore il me semble. Mais je pense que ce n'est pas seulement lié à ce que tu dis. II y a un article de Gilles Massardier ${ }^{2}$ qui analyse la manière dont les représentants de la nouvelle géographie et du GIP RECLUS, à un moment où il y avait beaucoup de moyens et de financements, ont co-forgé avec des fonctionnaires et des chargés de mission de la DATAR un langage commun, une analyse commune du territoire, et se sont rendus indispensables dans les réponses aux appels d'offre de la DATAR, dans les directions régionales et départementales.

AC : Et ce cadre limite largement le point de vue critique ; il peut certes contenir des éléments critiques, mais pas proposer de remise en cause fondamentale des rapports de pouvoir.

\footnotetext{
2 Gilles Massardier, 1996, "Les savants les plus "demandés". Expertise, compétences et multipositionnalité. Le cas des géographes dans la politique d'aménagement du territoire ", Politix, vol. 9, n³6, p. 163-180. G. Massardier est Maître de conférences en sciences politiques à Lyon 2, UMR 5206 "Triangle, Action, discours, pensée politique et économique ».
} 
S.W : Ce qui me frappe, c'est aussi la manière dont ces exigences ont percolé jusque dans les programmes scolaires qui ont beaucoup changé. Quand on regarde les derniers programmes scolaires, sur le développement durable, les territoires etc., maintenant, on ne traite plus systématiquement des inégalités Nord-Sud, on parle de différences culturelles. Et le fait que ce soit devenu aussi dominant dans l'enseignement secondaire, ça signifie qu'il faut tirer la sonnette d'alarme.

A.C : Et même dans l'enseignement supérieur, souvent. Enfin, pour ma part, ce que j'ai reçu pendant mes études ${ }^{3} n^{\prime}$ était pas très critique. Cela correspondait plutôt bien à la vision dominante d'un capitalisme qu'on n'interroge plus, ou alors, au mieux, pour dénoncer sa financiarisation.

S.W : Oui, le thème de la mondialisation a été catastrophique de ce point de vue alors que c'est une question centrale pour l'analyse du capitalisme. Mais ça dépend aussi des universités. II y a certaines d'universités où il y a toujours eu des gens prêts à mettre les pieds dans le plat.

A.C : II y a des gens qui sont critiques bien sûr, et l'enseignement que j'ai reçu en général pouvait être très bien, mais globalement, j'étais très frustrée de ce point de vue-là. Quand j'en parle avec mes collègues qui travaillent sur le Sud, ils n'avaient pas du tout la même impression cela dit. C'était assez segmenté.

S.W : C'était net à Paris 1 à l'époque ${ }^{4}$ oui, et aussi à l'École Normale ${ }^{5}$, du moins en géographie, mais pas en Histoire où il y existait une tradition forte, avec Yvon Thébert ou Annie Fourcaut par exemple.

\section{M.M : Quelles ambitions assignez-vous à ce colloque dans le mouvement de renouveau des pensées critiques?}

S.W : Tous les gens qui se posent ces questions sont les bienvenus pour en parler. II n'y a pas d'idée préconçue. Ce n'est pas limitatif. L'important c'est d'en parler pour donner un peu de visibilité à tous.

A.C : Nous souhaitions que les gens se rencontrent, se connaissent, qu'éventuellement un réseau commence à se créer. On le voit comme le début de quelque chose, mais ce n'est pas nous qui allons maîtriser ce processus. C'est pour cela que nous avons imaginé des ateliers-action où les gens seront invités à proposer des projets. Par exemple sur la question de l'intersectionnalité, qui est très peu travaillée en géographie par rapport à d'autres champs disciplinaires. Le but est de créer des liens, et ensuite de rester en contact pour aller plus loin. Plus loin qu'une simple critique de la ségrégation par exemple. Je n'ai jamais entendu un géographe dire que la ségrégation était une bonne chose; l'État condamne aussi la ségrégation, mais

\footnotetext{
${ }^{3}$ À l'ENS Fontenay-Saint-Cloud et à l'université Paris 1 et à Géographie-cités pour la thèse de doctorat.

${ }^{4}$ Dans les années 1990.

${ }^{5}$ À L'ENS Fontenay-Saint-Cloud et à l'université Paris 1, et à Géophile pour la thèse de doctorat.
} 
concrètement, il l'accompagne. II me semble qu'il faut qu'on pousse les gens à aller un peu plus loin.

S.W : Et puis je pense qu'il ne faut pas oublier de voir les liens entre le capitalisme et le patriarcat, les lectures culturalistes, essentialistes, ethnicistes sur l'intégration des minorités, des étrangers.... II faut remettre cela systématiquement en contexte par rapport au fonctionnement du capitalisme en se demandant à quoi ça se rattache matériellement, et comment les États-nations sont porteurs de ce système.

A.C: Oui, la critique du rôle de l'État dans le capitalisme est importante dans la littérature radicale anglophone. En France, la force du lien entre géographie et aménagement du territoire et la réponse à la commande publique limite la critique de l'État. Ou alors, c'est une critique de l'État néolibéral, comme si l'État keynésien n'était pas, lui aussi (même si sous une autre forme), un soutien au capitalisme et un outil de reproduction des rapports de pouvoir.

M.M : Apparemment, vous avez des sensibilités politiques différentes. Avez-vous néanmoins le sentiment de constituer un collectif scientifique, et comment avezvous travaillé collectivement pour préparer ce colloque?

S.W et A.C: Je ne crois pas que nous constituons un collectif. Nous travaillons par affinités individuelles et amicales autant que scientifiques. Nous travaillons tous les quatre sur des thématiques très variées. Nous participons à un projet de recherche sur l'Est francilien, en géographie sociale, qui porte sur la gentrification, la paupérisation de certains territoires, les trajectoires résidentielles, les mobilités quotidiennes [Anne], les migrations dans le périurbain lointain et la discrimination territoriale [Serge]. On s'efforce de trancher par rapport à l'approche classique de l'Est parisien en termes de transports, de politiques publiques. Respectivement, nous aimerions à terme travailler sur des questions de genre $[A C]$ et l'intersectionnalité à partir de travaux initiaux sur le travail féminin et subalterne [SW]. Quant aux deux autres organisateurs: Antoine [Fleury] travaille sur les politiques publiques portant sur les espaces publics à Paris, Istanbul et Berlin ; il est arrivé progressivement à une approche critique et s'intéresse de plus en plus aux inégalités d'accès à la centralité et aux espaces publics. Julien [Rebotier] travaille sur les risques urbains à Caracas et en ce moment sur une approche critique du développement durable et il tente de développer une approche marxiste des questions environnementales.

A.C : Que ce soit appelé à devenir un collectif, peut-être, mais il ne sera pas ancré à Marne-la-Vallée spécifiquement, même si le projet est porté par le laboratoire ACP ("Analyse Comparée des Pouvoirs »), essentiellement composé d'historiens qui ont tous une perspective critique. Reparler de questions anti-capitalistes et marxistes leur semble évident, même si je ne pense pas qu'ils se définissent eux-mêmes comme marxistes ou marxiens. Et puis, nous avons aussi ouvert à la pluridisciplinarité, en invitant notamment des sociologues, même si le colloque est essentiellement organisé par des géographes. 


\section{M.M : Quels types de contributions avez-vous reçues?}

SW et $A C$ : C'est très varié. II me semble qu'il y a une famille "villes, politiques urbaines", des contributions sur "Sud, développement ", très centrées sur la ruralité et sur l'Afrique, une partie " environnement et capitalisme " et une partie "genre ". Aucune thématique ne domine. Nous avons reçu des propositions en ethnologie, en sociologie et en sciences politiques; certaines contributions posent des questions théoriques et épistémologiques. Curieusement, il y a peu de choses sur les migrations et le travail des migrants; cette question semble encore traitée sous un angle très culturaliste finalement. Globalement, ce sont plutôt des jeunes chercheurs, même s'il y a aussi quelques chercheurs confirmés qui nous ont fait l'honneur de nous proposer des textes.

A.C: Ce qui nous frappe aussi, c'est que les rapports de domination ne sont pas forcément explicités dans les propositions. On les voit en filigrane. Nous allons faire des retours à certains contributeurs pour leur demander de préciser cet aspect. Mais on voit bien finalement que ce champ n'est pas mûr et que la majorité des propositions n'est pas strictement « dans l'appel ». Et puis, certains auteurs utilisent ces termes d'une autre manière que nous, ce qui sera une bonne occasion d'en discuter.

S.W : Ce sera aussi l'occasion de discuter de notions apparemment passe-partout en géographie, comme la notion de mobilité, dont on se rend compte en lisant ces propositions que, finalement, elle est une catégorie dominante. Idem pour les notions de mixité ou de développement durable, plus classiquement. Cela permettra de fourbir notre outillage critique.

M.M : Vous organisez un colloque qui se revendique en partie d'une approche marxiste. Quel est votre rapport à la praxis et à l'engagement politique ?

S.W : L'engagement, ce n'est pas un attendu pour les participants au colloque. L'appel ouvre aux militants, afin que cette question puisse être abordée, mais ce n'est pas un attendu a priori.

A.C : Personne parmi nous ne se dit marxiste je crois. Même si cela fait partie de notre répertoire, cela me paraît trop restrictif. Nous nous revendiquons plutôt d'une approche matérialiste, d'une géographie des fondements matériels des inégalités, pas seulement de classes. Par ailleurs, les organisateurs du colloque sont inégalement engagés politiquement et ont des rapports très variées au militantisme. De mon côté, j'ai milité et je suis aujourd'hui en retrait. Mais ma motivation pour organiser ce colloque est autant scientifique et théorique que militante. Ce colloque correspond aussi à une envie de faire quelque chose de radical dans le monde universitaire, comme j'ai pu le faire dans le monde militant. Bien sûr, organiser un colloque ne suffit pas à changer le monde et le militantisme académique n'est pas un vrai engagement ; ça ne remplace pas un engagement dans des organisations politiques ou syndicales. Et de ce point de vue, les modalités de notre travail sont problématiques, car en ce qui me concerne, j'ai du mal à garder un lien avec l'engagement. Mais ce réseau, s'il se 
développe, et si les gens sont motivés, j'aimerais bien qu'il permette, à terme, une forme d'engagement, en encourageant les géographes radicaux à s'exprimer dans la sphère politique, pas à tout bout de champ, mais sur certains points sur lesquels ils ont des choses à dire.

M.M : Vous voyez à travers ce colloque un acte militant. Mais comment définiriezvous plus précisément la nature de cet engagement ? Avez-vous par exemple prévu des espaces d'échange dédiés à des contacts avec les milieux militants, la société civile ? Je pense à vos ateliers-action.

A.C : Les ateliers-actions n'auront pas a priori de contenu militant. Ils visent en fait à susciter des prolongements académiques à ce colloque (séminaire, publications...). L'idée de faire de la recherche-action au sens militant ne me semble pas mûre pour l'instant. Je serais ravie que ça se développe, mais c'est plutôt une perspective à long terme et quelque chose à développer collectivement.

S.W : Et puis ce seront des espaces pour discuter de méthodes, de manières de travailler, non institutionnelles ou non conventionnelles, de nouvelles manières de faire.

A.C: Oui, c'est comme réfléchir aux rapports de domination au sein du monde académique. Là aussi beaucoup de choses ont déjà été dites. En revanche, c'est quelque chose qui est toujours à refaire dans des collectifs, de reposer constamment ces questions, surtout quand on intègre des jeunes.

S.W : De mon côté, ce qui m'intéressait c'était aussi la question de l'enseignement de la géographie, pas seulement à l'université, où je fais mes cours de manière très libre et en abordant des questions politiques. Mais pour avoir siégé au jury du CAPES pendant plusieurs années et avoir été proche de la question de la géographie enseignée, je sais qu'il y a beaucoup d'enseignants du secondaire qui sont très demandeurs de pouvoir enseigner autre chose que ce que les programmes récents leur imposent. J'aurais aimé que nous ayons un atelier et des contributions sur ce sujet, et nous en avions discuté. Mais ça n'a pas été le cas. Et j'ai aussi l'idée de faire un manuel critique.

A.C : Nous allons aussi inviter des militants et diffuser l'information dans des cercles militants, et on verra si des gens viennent et si cela prend. Nous avons eu très peu de contributions de militants. Mais c'est vrai que ce colloque reste très classique dans sa forme et vise à faire bouger les lignes à l'intérieur du monde académique. Pour I'instant, nous n'avons pas non plus encore trouvé de moyen d'interagir avec le monde militant car il y a peu de collectifs constitués sur des questions liées à l'espace. Notre engagement se fait donc surtout individuellement, en fonction de nos intérêts personnels.

S.W : Oui, je suis pour ma part en contact avec des collectifs et des associations sur les droits des migrants où j'interviens régulièrement, mais toujours en tant que scientifique, pour discuter avec des militants, faire des journées de formation... Ces 
gens sont très marqués par l'approche "droits de l'homme " et éloignés de la géographie. Je pense qu'il est intéressant de réfléchir avec eux à la dimension politique de la question des droits de l'homme.

A.C : Dans le champ urbain, aux États-Unis, un mouvement pour le droit à la ville s'est constitué récemment. Si cela existait en France, il aurait été plus évident de faire le lien avec eux, mais ici, c'est très éclaté. II n'y a pas de mouvement facilement identifiable avec lequel faire le lien. Cela dit, pour moi, il faut que ce lien soit fait à un moment. Mais pour l'instant, je crois qu'on a d'abord besoin de se rassembler dans le monde académique.

S.W : Nous enverrons aussi le programme à des listes de diffusion d'enseignants du secondaire.

M.M : Il existe un débat sur le sens politique et institutionnel de l'approche critique, parfois taxée de constituer autant une posture carriériste qu'un positionnement scientifique, notamment dans le monde anglophone où elle dispose d'une grande visibilité académique. Qu'en pensez-vous ? Quelles leçons tirez-vous de ce point de vue des expériences nord-américaines ou britanniques?

S.W : Je n'ai pas l'impression qu'en géographie, ce soit une niche pour la carrière.

\section{A.C : Ce n'est pas très porteur.}

S.W : Je dirais que ce n'est ni handicapant, ni quoi que ce soit. Notamment, quand on regarde passer les thèses et les candidatures, je ne crois pas...

A.C : Je pense que ça peut l'être, quand on commence une thèse par exemple. Et puis cela dépend des postes, des universités, des directeurs. Là aussi, c'est très éclaté. Mais des géographes radicaux qui sont des ténors académiques, je ne vois pas trop cela en France. En ce qui nous concerne, nous sommes tous de jeunes chercheurs, même dans le comité scientifique, et nous ne nous sentons pas vraiment concernés par ces débats, même si j'aimerais aussi que nous ayons cette réflexion sur les pratiques de pouvoir au sein de l'université. Nous sommes tous très jeunes en poste, et pour nous c'est certainement un chantier que de réfléchir à nos pratiques, pour éviter la perpétuation de pratiques par exemple patriarcales que reproduisent des gens qui se disent radicaux.

S.W : Je suis plus sceptique que toi sur la capacité à contrôler nos pratiques institutionnelles. Et puis, cela dépend beaucoup des universités. Nous avons une expérience très positive de ce point de vue à Marne-la-Vallée. Les choses sont sans doute différentes dans des universités plus grandes et nous pourrons sans doute en discuter avec les participants au colloque qui viennent d'institutions diverses. Mais pour moi, en tout cas, l'approche critique ne relève pas d'une posture. Elle constitue un véritable défi scientifique : on a beau essayer de faire tenir les raisonnements, si on n'a pas cette question du fondement matériel, ça ne marche pas. 
A.C : La motivation la plus forte est donc scientifique. Là-dessus je crois qu'on est d'accord. Elle est de lire des choses qui me convainquent et me paraissent expliquer vraiment le monde. Car il y a quelque chose dans l'idée de la science qui relève du pouvoir explicatif. C'est vrai que ce colloque vient au départ d'une rencontre avec $\mathrm{N}$. Smith, à New York, que je l'avais lu avant, et qu'au début de ma thèse, c'est ce qui m'a convaincu que ça valait la peine de faire une thèse en géographie. Harvey, je l'ai lu beaucoup plus tard. En les lisant, je trouvais claire et convaincante cette manière de faire le lien avec le capitalisme. Donc c'est une motivation intellectuelle. Ce qui me frustre intellectuellement dans beaucoup d'approches géographiques, et même parfois de géographies critiques, notamment culturelles, c'est que ça ne marche pas jusqu'au bout. II manque quelque chose. Mon plus grand plaisir intellectuel, je l'ai eu en lisant des textes radicaux, surtout anglophones c'est vrai, ou chez des sociologues. Et puis, dans le monde anglophone, ce n'est pas si homogène. D. Harvey n'a jamais été militant, contrairement à N. Smith qui est très engagé. Ce n'est pas le même rapport à l'académie. Par ailleurs, cette structuration en sous-champs, ce n'est pas seulement le fait des géographes radicaux. C'est aussi un effet du système pour leur faire une place à part, pour qu'ils ne contaminent pas tout le reste. Et puis il y a certainement des spécificités dans l'organisation des universités anglo-saxonnes qui font que cela ne pourrait pas se reproduire tel quel en France. Cela dit, si on regarde l'économie hétérodoxe en France, on laisse parler des gens qui tiennent des discours très radicaux, et c'est une stratégie ; c'est le système capitaliste qui permet ça. Plutôt que de contrer et de faire de la censure.

S.W : Ce qui m'a fait réagir aussi sur la nécessité d'une approche radicale, c'est le nombre d'approches théoriques en géographie qui sont très englobantes, et tentent de rendre compte du fonctionnement de tout; c'est une sorte de lissage, d'aplatissement et on ne sait plus du tout quels sont les phénomènes de causalité. II y a aussi eu une période où le succès de systèmes de pensées comme celui de Giddens en géographie m'a frappé. Finalement, ça marche comme ça, donc tout va rentrer dans des petites cases, et ce n'est plus la peine de bouger. Et pour moi, une attitude théorique, c'est aussi d'empêcher de tourner en rond, de permettre de voir de quoi je suis prisonnier. C'est le principe du raisonnement critique en général. De quoi et en quoi suis-je victime, sans en avoir conscience ? Une remise en question.

A.C : Inversement, Harvey que j'ai lu vraiment tard, propose aussi une explication englobante que je trouve stimulante.

S.W : Ce n'est pas qu'elle est englobante, c'est qu'elle est fonctionnelle pour tout. C'est une bonne clé.

A.C : C'est vrai que je suis attirée par ce type de systèmes explicatifs, qui restent toujours à approfondir cela dit. Un travail sur un point précis, on peut trouver que ça tient, mais souvent, ça se relie mal au reste. Les travaux de D. Harvey permettent d'analyser une situation concrète locale tout en faisant le lien avec le global. C'est ce que j'essaye de faire quand je le peux. 
M.M : Les approches critiques sont moins structurées institutionnellement en France. Selon vous, est-ce lié à l'importance de l'approche empirique dans la géographie française ? Peut-on imaginer l'émergence d'une pensée critique à la française, et qu'est-ce qui la distinguerait selon vous ? Pourrait-elle par exemple s'appuyer sur cette tradition d'empirisme?

\section{S.W : Je préfèrerais répondre après le colloque!}

A.C: "À la française " me gêne un peu. Que ce soit français, ça ne me semble pas important. Je préfère qu'on soit bien reliés aux anglophones. L'idée n'est pas de faire quelque chose de franco-français, centré sur la France, mais de faire émerger quelque chose en France, parce que selon moi nous sommes à la traîne sur ces questions par rapport à d'autres disciplines et par rapport à d'autres pays.

S.W : Oui, par exemple les économistes marxistes en France sont minoritaires, mais ils existent. Les sociologues aussi, qui ont Bourdieu, une source d'inspiration majeure d'ailleurs dans les pays anglophones. "À la française ", je n'y crois pas trop, mais « en France ", je dirais oui. Par ailleurs en France, les approches qui sont les plus proches des perspectives radicales, ou les plus attentives à la dimension matérielle et historique des phénomènes, ce sont des études très empiriques. Beaucoup de géographes en France, en particulier mais pas seulement ceux qui travaillent sur le Sud, font des études très empiriques. Dans les colloques internationaux, il n'y pas de communications de géographes français qui soient strictement théoriques. On s'écoute donc différemment en France, quel que soit notre positionnement. Mais je ne dirais pas que cela a freiné le développement des pensées critiques. Je dirais plutôt que certaines grilles de lecture, un peu clés en mains, n'ont pas pris (je pense au débat sur " en finir avec les études post-coloniales $\left.{ }^{6}\right)$. Je trouve ça très stimulant : tout d'un coup, on a vu des chercheurs français (des historiens, des sociologues...) remettre en cause des approches critiques (comme la critique post-coloniale); ils nous rappellent qu'il faut aller dans les archives, sur le terrain et voir ce qui se passe. Par exemple, S. Jaglin, qui ne revendique d'appartenir à un courant de pensée en particulier, ni critique, ni post-moderne, ni de géographie radicale, est sur le terrain ; elle a d'ailleurs contre argumenté sur notre projet de colloque de manière très stimulante, en me disant qu'on datait un peu avec D. Harvey. Et le fait que de grands courants n'aient pas pris en France, ça me semble un avantage. Mais de l'autre côté, la plupart des études qui sont attentives aux dimensions matérielles ne se revendiquent pas du marxisme ou d'une école de pensée critique pour lesquelles Marx n'est pas une référence explicite, et cela pose aussi problème.

A.C : Sur l'enjeu de la transmission, notamment aux jeunes chercheurs, je trouve quand même que ça pose problème. Par ailleurs, on n'a pas le même poids si on n'est pas structuré collectivement; ça fait partie de l'approche radicale que de croire à

\footnotetext{
${ }^{6}$ Allusion aux attaques de J-F. Bayart contre le succès des études post-coloniales anglophones (Bayart JF., 2009, "En finir avec les études post-coloniales ", Le Débat, n¹54, p. 119-140 et Bayart J-F., 2010, Les études postcoloniales. Un carnaval académique, Paris, Karthala), et plus largement au débat sur les postcolonial studies en France, important au milieu des années 2000.
} 
I'action collective. Je me souviens que N. Smith m'a dit avoir été frustré quand il a tenté de contacter Y. Lacoste, qu'il percevait comme un géographe radical en France, et que ce dernier ne lui a pas répondu; il m'avait expliqué qu'il lui semblait que les universités étatsuniennes étaient finalement moins liées qu'en France à la commande publique, à l'aménagement et à l'institution scolaire. Ce serait donc moins un problème de rapport à l'empirie que de structures institutionnelles qui laisseraient peu de place à la critique de l'État.

S.W : Je suis d'accord. Mais n'oublions pas que le monde de la recherche est aussi très individualiste. Construire en commun, ça me semble commencer par discuter et interroger certaines catégories, notamment lors de ce colloque.

\section{M.M : N'avez-vous pas le sentiment, en invitant en séance plénière des vedettes de la géographie anglophone (D. Mitchell, N. Smith, J. Ribot ${ }^{7}$ ), de renforcer un processus de domination académique (culturel, politique, institutionnel, linguistique...) ?}

A.C : Nous avons invité des sociologues français et des géographes étrangers. Outre D. Mitchell et N. Smith, nous avons aussi invité Christine Delphy, Jean-Pierre Garnier, Jesse Ribot et Pierpaolo Mudu ${ }^{8}$. Nous avons chacun proposé un ou plusieurs invités parce qu'ils étaient une référence scientifique pour nous et notre travail, pas parce qu'ils étaient des vedettes.

S.W : Et Pierpaolo Mudu est même une anti-vedette. Par la force des choses, il n'appartient réellement à aucune institution universitaire. C'est le plus cohérent dans son positionnement de ce point de vue, à mon sens. II est très demandé et très reconnu et fait d'énormes enquêtes qui ont un fort impact politique. Mais il a choisi de travailler dans une certaine précarité qui lui donne aussi une certaine liberté.

A.C : Smith et Mitchell, comme souvent les Étatsuniens, sont très accessibles. J'ai rencontré N. Smith à l'occasion d'un voyage à New York il y a deux-trois ans, après l'avoir simplement contacté par mail en lui disant que j'avais fait ma thèse sur la

\footnotetext{
${ }^{7}$ Don Mitchell, Professeur à Syracuse University (New York) est un géographe radical spécialiste des questions de droit à la ville, de justice et de mouvements sociaux en ville. Jesse Ribot, Maître de conférences à l'Université Urbana-Champaign est un géographe diplômé de Berkeley; ses travaux portent sur l'accès aux ressources naturelles et la vulnérabilité des ménages face au changement climatique ; son parcours l'a conduit du MIT, au Max Planck Institute, à Harvard et à Yale. II a produit de nombreux rapports pour la Banque mondiale et les Nations-Unies. N. Smith, diplômé de l'Université John Hopkins (où il fut l'élève de D. Harvey), est Professeur d'anthropologie et de géographie à la City University de New York ; il a enseigné à l'université de Pennsylvanie, à Columbia et à Rutgers Universities et produit notamment des travaux empiriques et théoriques sur la gentrification en Amérique du Nord qui soulignent l'importance des mécanismes financiers et immobiliers dans ce processus.

${ }^{8}$ Christine Delphy est Directrice de recherches émérite au CNRS, sociologue rattachée à l'UMR "Triangle » et à l'École Normale Supérieure de Lyon, spécialiste des questions de genre et représentante du courant du féminisme matérialiste, co-fondatrice et Directrice de la revue Nouvelles Questions Féministes ; Jean-Pierre Garnier est sociologue et urbaniste, ingénieur de recherche retraité et militant politique ; Pierpaolo Mudu est professeur associé en études urbaines, Université de Washington Tacoma (États-Unis), spécialiste des questions de santé et de migrations ; Jesse Ribot, malgré son intérêt pour cette initiative, n'a pas pu se libérer finalement et ne sera donc pas présent.
} 
gentrification. Cela m'a fait très plaisir de le rencontrer. C'est quelqu'un de très sympa, rien qui permet de soupçonner qu'il est si connu. C'est un activiste, son bureau est couvert d'affiches militantes. Et comme chacun.e des organisateurs du colloque devait proposer une ou plusieurs personnes qui l'avaient marqué.e dans ses recherches, son nom s'est imposé à moi. C'est après coup, quand on nous a dit que nous n'avions retenu que des géographes anglophones ou des sociologues français, que je me suis rendue compte que cela pouvait être très mal perçu. On a fait le choix, en tout cas, de ne pas inviter D. Harvey. II faisait vraiment trop vedette. Et puis l'idée, c'était d'avoir des liens académiques concrets avec des chercheurs encore actifs, de pouvoir à terme envoyer des doctorants, etc.

S.W : L'élément de l'appel qui a gêné certaines personnes je crois, c'est qu'on les a annoncés et programmés d'emblée. Mais ce sont tous des gens qu'on a choisis pour la portée théorique de leur pensée. L'idée est donc bien au contraire de faire émerger un dialogue en France, afin qu'on ne reste pas dominés justement, et qu'on apporte des choses à ce champ-là. Cette confrontation est importante. II faut se demander ce qui se passe ailleurs. 